\title{
Quaking I-5 protein inhibits invasion and migration of kidney renal clear cell carcinoma via inhibiting epithelial-mesenchymal transition suppression through the regulation of microRNA 200c
}

\author{
Ruili Zhang ${ }^{1}$, Wenguang Wang ${ }^{2}$, Ainiwaer Aimudula ${ }^{1}$, Songmei Lu ${ }^{1}$, Pengfei Lu ${ }^{1}$, Remila Aihaiti ${ }^{1}$, \\ Yongxing Bao ${ }^{1}$ \\ ${ }^{1}$ Cancer Center, First Affiliated Hospital of Xinjiang Medical University, Urumqi, China; ${ }^{2}$ Department of Urology, First Affiliated Hospital of \\ Xinjiang Medical University, Urumqi, China \\ Contributions: (I) Conception and design: All authors; (II) Administrative support: None; (III) Provision of study materials or patients: None; (IV) \\ Collection and assembly of data: R Zhang, W Wang; (V) Data analysis and interpretation: A Aimudula, S Lu; (VI) Manuscript writing: All authors; (VII) \\ Final approval of manuscript: All authors. \\ Correspondence to: Ruili Zhang. Cancer Center, First Affiliated Hospital of Xinjiang Medical University, Urumqi 830054, China. \\ Email: 58419098@qq.com.
}

Background: It has been demonstrated that quaking I-5 protein (QKI-5) plays crucial roles in the metastasis of various kinds of cancers. However, the function and mechanism of QKI-5 in kidney renal clear cell carcinoma (KIRC) metastasis remains unclear. Therefore, this study aimed to explore the mechanism of QKI-5 in the metastasis of KIRC.

Methods: The expression of QKI-5 was detected using real-time quantitative reverse transcription polymerase chain reaction (qRT-PCR) and western blot in KIRC tissues and different cell lines. Immunohistochemical staining was used to detect the quantity of QKI-5 in primary and metastases of KIRC. Cell migration and invasion were measured using wound healing and transwell assays respectively. The quantity of epithelial mesenchymal transition marker proteins was detected using western blot and immunofluorescence staining. The interaction of QKI-5 via microRNA 200c (miR-200c) was confirmed using dual luciferase reporter assay.

Results: Although QKI-5 was significantly more likely to be downregulated in KIRC tissues than that in normal Kidney tissues, it was dramatically elevated in metastatic KIRC tumors. Upregulation of QKI-5 promoted cell migration and invasion and elevated the expression of epithelial-mesenchymal transition (EMT) marker proteins, including vimentin, snail and slug, while it was downregulated for E-cadherin. Furthermore, a dual luciferase reporter assay demonstrated that QKI-5 was a direct target of miR-200c, and that miR-200c could reverse the effect of QKI-5 on cell migration, invasion, and expression of EMT marker proteins.

Conclusions: Our results revealed that downregulation of QKI-5 by miR-200c attenuated KIRC migration and invasion via the EMT process, indicating that QKI-5 may be a potential therapeutic target and a key indicator of KIRC progression.

Keywords: miR-200c; kidney renal clear cell carcinoma (KIRC); QKI-5; epithelial-mesenchymal transition (EMT)

Submitted Aug 26, 2021. Accepted for publication Oct 20, 2021.

doi: $10.21037 /$ tau-21-833

View this article at: https://dx.doi.org/10.21037/tau-21-833 


\section{Introduction}

Renal cell carcinoma (RCC), which is derived from renal tubular epithelial cells, ranks second among all types of urinary system malignancies (1). kidney renal clear cell carcinoma (KIRC) is the most common histological type of renal cell carcinoma, accounting for about $70-80 \%$ of all RCCs with a very high mortality rate of about $40 \%$. Although significant progress in the diagnosis and treatment of KIRC has been made, the 5-year survival rate of KIRC is less than $10 \%$ owing to high recurrence and metastasis rates $(2,3)$. Therefore, research on the mechanism of KIRC metastasis is needed urgently.

RNA-binding protein quaking (QKI) belongs to the conserved signal transduction and activation of RNA (STAR) protein family. It is one of the QKI protein isoforms, which are found mostly in the nucleus and have been proven to play a complex role in tumorigenesis and metastasis in different cancers. It has been demonstrated that QKI-5 suppresses epithelial-mesenchymal transition (EMT) and tumor growth in prostate cancer (4) and inhibits the aggressiveness of lung cancer cells through the inhibiting $\beta$-catenin signaling pathway (5). In oral squamous cell carcinoma, QKI-5 inhibits cell proliferation, migration, and invasion in vitro and in vivo (6). However, QKI-5 has been found to promote cell proliferation, invasion, and EMT in esophageal squamous cell carcinoma (7) and in lung cancer, nanoparticle-mediated RNA interference of endothelial QKI expression potently inhibits metastasis (8). In our past research, we investigated the role of QKI in kidney tumorigenesis. We demonstrated that QKI inhibits kidney cancer cell growth in part through the QKI-directed upregulation of RASA1 by directly binding to its QRE region(QKI response element; ACUAAY[N1-20]UAAY) (9), we found that mostly the relative expression level of QKI5 was significantly lower in the tumour tissues than it was in their noncancerous counterparts, but once the tumor tissue invades into extrarenal adipose tissue, the expression level of QKI-5 will be significantly higher, which suggest QKI-5 may influence tumor invasion, since the function and mechanism of QKI-5 in KIRC metastasis remains unclear, it is necessary to do further investigations of QKI5 for better understanding the mechanisms of kidney tumor invasion and metastasis.

EMT is a reversible cellular process between epithelial and mesenchymal states (10). To date, increasing evidence has demonstrated that EMT contributes to tumor progression in various cancers, especially in tumor invasion and metastasis (11). EMT is governed by networks of transcriptional and post-transcriptional mechanisms, such as extracellular matrix, adhesion molecules, cytoskeletal proteins transcription factors, and noncoding RNAs (12). In fact, several studies have been demonstrated that QKI5 affects the EMT process during tumorigenesis. It has been reported that QKI broadly promotes mesenchymal splicing patterns (13), and that QKI-5 expression changes are regulated by microRNAs (miRNAs), such as miR-196b5p, miR-200/375, miR-574-5p, and miR-221 (14-17). Of these, miR-200c belongs to the miR-200 family and has been demonstrated to be involved in tumor progression by regulating the EMT process in several cancers, such as prostate and colorectal cancer (18-20). In addition, we showed that QKI-5 is dramatically elevated in metastatic KIRC tumors compared to primary KIRC tumors, demonstrating that QKI-5 might be regulated by miR-200c, thus contributing to the metastasis of KIRC. Therefore, we aim to clarify the effects and the mechanism of QKI-5 on KIRC cell invasion, migration, and EMT that contributes to tumor development regulated by miR-200c.

We present the following article in accordance with the MDAR reporting checklist (available at https://dx.doi. org/10.21037/tau-21-833).

\section{Methods}

\section{Clinical information}

Twenty cases of Matched KIRC tissues and corresponding tumour-adjacent, morphologically normal tissues were collected from 20 KIRC patients seen at the First Affiliated Hospital of Xinjiang Medical University from 2010 to 2016. All the KIRC tissue and corresponding tumour-adjacent, morphologically normal tissues samples were then immediately flash-frozen in liquid nitrogen at $-80{ }^{\circ} \mathrm{C}$. Another 20 matched primary KIRC tissues and metastatic KIRC tissues used for immunohistochemical (IHC) staining were obtained from the First Affiliated Hospital of Xinjiang Medical University from 2010 to 2018 .

All procedures performed in this study involving human participants were in accordance with the Declaration of Helsinki (as revised in 2013). The project has been approved by the ethics committee of the First Affiliated Hospital of Xinjiang Medical University (No.: 20180213-09), and all of the participants have provided their informed consent. 


\section{QKI-5 lentivirus construction, cell culture, and treatment}

Lentivirus with overexpressing QKI-5 sequences and shQKI-5 sequences was created into pLenO-DCE and pLenR-GPH vectors, respectively. Then, vectors were used to obtain overexpressing QKI-5 and suppressing QKI-5 lentivirus according to the lentivirus packaging instructions. Human clear cell renal cell carcinoma cell lines 786-O and Caki-2, human kidney adenocarcinoma cell line ACHN, human kidney cancer cell lines A-498 and SN12C, human renal papillary cell line SKRC-38, and human renal tubular epithelial cell line HK-2 were purchased from Shanghai Institute of cell biology After cell lines recovery, cells were cultured in 90\% Dulbecco's modified Eagle's medium (DMEM, 12430054, Gibco, Waltham, MA, USA) supplemented with $10 \%$ of fetal bovine serum (FBS, Gibco, Waltham, MA, USA), $100 \mathrm{U} / \mathrm{mL}$ penicillin and $100 \mu \mathrm{g} / \mathrm{mL}$ streptomycin at $37{ }^{\circ} \mathrm{C}$ in a $5 \% \mathrm{CO}_{2}$-contained incubator under 95\% saturation humidity. For overexpressing QKI-5 and suppressing QKI-5 lentivirus and transfection, 786-O, ACHN, or Caki-2 cells were seeded in a six-well plate until confluence was reached at $70 \%$ to $90 \%$. Then, overexpressing QKI-5 or suppressing QKI-5 lentivirus and the miR-200c NC (non-specific control) and mimics were transiently transfected into 786-O or Caki-2 cells and cultured for $24 \mathrm{~h}$. Next, cells were used for further analysis. The miR-200c and NC mimics were synthesized using RiboBio Co. Ltd. (Guangzhou, China), and sequences were listed at Table S1.

\section{Migration and invasion assays}

Cell migration abilities and Cell invasion abilities of 786-O, ACHN, and Caki-2 cells were evaluated using wound healing assays and transwell assays according to previous reports. For wound healing assays, Seed cells in 6 well plates overnight and culture until confluent. Using a (blue) pipette tip make a straight scratch, simulating a wound. Finally discard the media from the wells and add fresh medium to wells. For transwell assays. Briefly, a total of $5 \times 10^{4}$ suspended $786-\mathrm{O}$, ACHN, or Caki-2 cells with serum-free media were placed into the upper part of an insert, and subsequently medium with $10 \%$ of FBS was added to the lower part. Once the cells had migrated through the membrane after incubation for $24 \mathrm{~h}$, they were fixed with $100 \%$ methanol and stained with $0.1 \%$ crystal violet. The invasive stained cells and the fixed cells were observed and counted using a light microscope (Olympus,
Tokyo, Japan). Three experiments were performed independently.

\section{Quantitative real-time reverse transcription-polymerase chain reaction ( $q$ RT-PCR)}

Total RNA was extracted from cells or tumor tissues using Trizol reagent (Takara, Dalian, China). Two ug of RNA were used to synthesize cDNA using the Bestar ${ }^{\mathrm{TM}}$ qPCR RT Kit (2221, DBI Bioscience, Newark, DE, USA) and Bestar $^{\text {TM }}$ miRNA qPCR RT kit (2221, DBI, Bioscience, Newark, DE, USA). Relative QKI-5 or miR-200c levels were measured using qRT-PCR with a $20 \mu \mathrm{L}$ reaction system, including $10 \mu \mathrm{L}$ SYBR Mix (Thermo fisher, Waltham, MA, USA), $0.5 \mu \mathrm{L}$ of each primer $(10 \mu \mathrm{M}), 2 \mu \mathrm{L}$, cDNA template, and $7 \mu \mathrm{L}$ RNase ddH2O. ABI PRISM 7500 real-time PCR system (Applied Biosystems, Carlsbad, CA, USA) was used to perform the PCR using the following processes: $95^{\circ} \mathrm{C}, 5 \mathrm{~min}$; followed by 40 cycles of $94^{\circ} \mathrm{C}, 30$ $\mathrm{s}, 60^{\circ} \mathrm{C}, 40 \mathrm{~s}$ and $72{ }^{\circ} \mathrm{C}, 15 \mathrm{~s}$. As the internal reference for miR-200c and QKI-5, U6 and glyceraldehyde 3-phosphate dehydrogenase (GAPDH) were used. The fold change of QKI-5 and miR-200c expression was calculated using the $2^{-\Delta \Delta \mathrm{Ct}}$ method for at least three biological repeats for each sample. All the primer sequences used are listed in Table S1.

\section{Western blot}

Western blot analysis was performed according to the previous report. Briefly, total proteins from 786-O, ACHN, and Caki-2 cells or tumor tissues were extracted using RIPA Lysis buffer (E-BC-R327, Elabscience, Houston, TX, USA) and then the protein concentration was measured using a bicinchoninic acid (BCA) protein assay kit. For western blot, $20 \mathrm{~g}$ of protein with protein loading buffer were boiled at $100{ }^{\circ} \mathrm{C}$ for $10 \mathrm{~min}$ for each sample, followed by separation in $10-12 \%$ sodium dodecyl sulphate-polyacrylamide gel electrophoresis (SDS-PAGE). Then, 5\% lipid-free milk buffer were used to block the membranes with blots for $2 \mathrm{~h}$ at room temperature after transferring onto polyvinylidene fluoride (PVDF) membranes (Millipore, Billerica, MA, USA), incubated with primary antibody against QKI-5 (ab126742, 1:2,000, Abcam, Cambridge, United Kingdom), E-cadherin (ab40772, 1:1,000, Abcam), vimentin (ab92547, 1:1,000, Abcam, Cambridge, United Kingdom), $\beta$-catenin (ab32572, 1:2,000, Abcam, Cambridge, United Kingdom), SNAIL (ab216347, 1:1,000, Abcam, Cambridge, United Kingdom), SLUG (ab51772, 1:1,000, Abcam), and GAPDH 
(1:2,000, Rabbit polyclonal, Abcam, Cambridge, United Kingdom) overnight at $4{ }^{\circ} \mathrm{C}$. Finally, protein signals were detected using enhanced chemiluminescence (ECL) reagent (Thermo Scientific, Waltham, MA, USA) based on the manufacturer's instructions. After incubation with secondary antibody anti-mouse immunoglobulin (IgG, ab205719, 1:20,000, Abcam, Cambridge, UK) for 2-3 hours.

\section{Immunobistochemistry}

Tumor tissues from different groups were immediately dissected and fixed in $10 \%$ buffered formalin and processed in a paraffin tissue processing machine. Sections of $7 \mu \mathrm{m}$ thickness were stained with haematoxylin and eosin (H\&E, G1005, Servicebio, Wuhan, China). Briefly, the specimen was fixed using $10 \%$ formaldehyde, and $4 \mu \mathrm{m}$ serial sections were generated after embedding in paraffin. Slices were inactivated in $3 \%$ catalase for $15 \mathrm{~min}$ after having been placed in an oven for $1 \mathrm{~h}$ at $60^{\circ} \mathrm{C}$. Then, the sections were placed at $37{ }^{\circ} \mathrm{C}$ and blocked using $5 \%$ bovine serum albumin (BSA) for $30 \mathrm{~min}$. Subsequently, the tumor sections were incubated with KI67 Rabbit Polyclonal antibody (27309-1-AP, 1:500, Proteintech, Rosemont, IL, USA) at $4{ }^{\circ} \mathrm{C}$ overnight followed by incubation with biotinylated goat anti-rabbit IgG (ab6721, 1:1,000, Abcam, Cambridge, UK) at $37^{\circ} \mathrm{C}$ for $30 \mathrm{~min}$ after having been washed with $0.2 \mathrm{~mol} / \mathrm{L}$ PBS solution (PH 7.4). DAB (K5007, DAKO, Glostrup, Denmark) was used for color rendering, and then the sections were stored at room temperature for $8 \mathrm{~min}$ in a dark room. Finally, sections were observed using a XSP-36 microscope (Boshida Optical Co., Ltd, Shenzhen, China).

\section{Bioinformatics analysis}

Target miRNAs of QKI-5 were predicted using Targetscan 7.2 (http://www.targetscan.org/vert_72/), Starbase 2.0 (http://starbase.sysu.edu.cn/) and miRWalk (http://mirwalk. umm.uni-heidelberg.de/). online software. Then, QKI-5 and the miRNA expression data were obtained from The Cancer Genome Atlas Program database (TCGA, https:// www.cancer.gov/about-nci/organization/ccg/research/ structural-genomics/tcga). The correlations between QKI5 and miRNAs were calculated with Spearman tests using R software (The R Foundation of Statistical Computing, Vienna, Austria). The overall survival for ccRCC patients in $\mathrm{T} 3$ or T4 status was determined using the log-rank test.

\section{Dual luciferase reporter assay}

TargetScan 7.2 (http://www.targetscan.org) was used to predict the putative targets of miR-200c with 3'UTR of QKI-5. The interaction between miR-200c and 3'UTR of QKI-5 was evaluated using the Dual-Luciferase Reporter Assay System (Promega, Madison, WI, USA) as instructed by the manufacturer. For the 3'UTR of QKI-5, wild and mutant sites were constructed and subcloned into pmirGLO vector. Then, MiR-200c mimic/NC and 3'-UTR of QKI-5 were co-transfected into 293T cells using the Lipofectamine $^{\mathrm{TM}} 2000$ transfection reagents (Cat. \#52887, Invitrogen, Carlsbad, CA, USA). Cells were washed twice with phosphate buffered saline and lysed using the passive lysis buffer at 48 hours after transfection. Luciferase activity was evaluated using the Dual-Luciferase Reporter Assay System (Promega, Madison, WI, USA) according to the manufacturer's instruction. Primers used for vector construction are listed in Table S1.

\section{Statistical analysis}

All data are shown as means \pm standard deviations $(\mathrm{SD})$ and were analyzed using SPSS 21.0 (SPSS, Inc., Chicago, IL, USA) software. One-way analysis of variance (ANOVA) was followed by Dunnett's multiple comparison tests and student's $t$-tests to assess the statistical significance of the differences between treatment groups. $\mathrm{P}<0.05$ was considered as indicating statistical significance.

\section{Results}

\section{Characteristic of QKI-5 in KIRC}

It has been proven that QKI-5 is downregulated in KIRC samples corresponding to noncancerous normal tissues. Consistent with these results, qRT-PCR and western blot analysis showed that the expression of QKI-5 was significantly downregulated in KIRC tissues compared to normal kidney tissues (Figure 1A-1C). However, we found that the expression of QKI-5 was dramatically elevated in metastatic KIRC compared to primary KIRC using immunohistochemical staining (Figure 1D), suggesting that QKI might play crucial roles in the metastasis of KIRC. Further analysis showed that lower QKI-5 expression level demonstrated better overall survival during both the T3 and T4 periods of KIRC (Figure 1E,1F). 

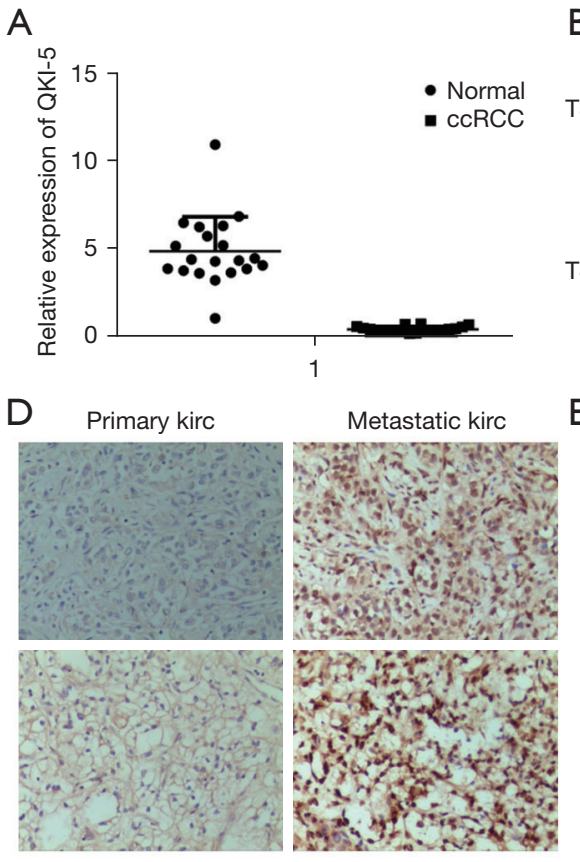

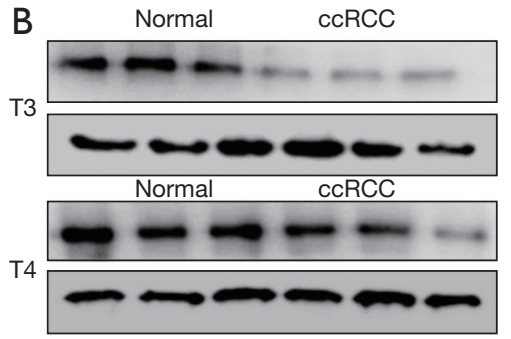

$\mathrm{E}$

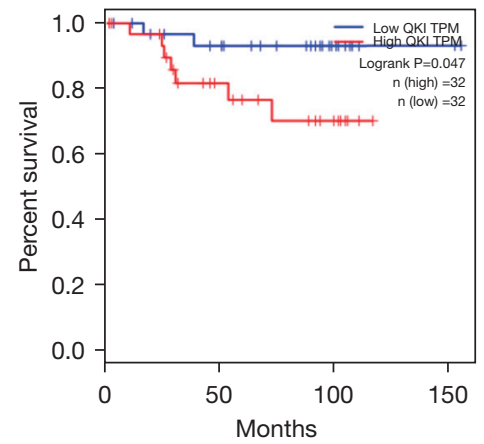

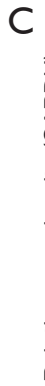

$\mathrm{F}$
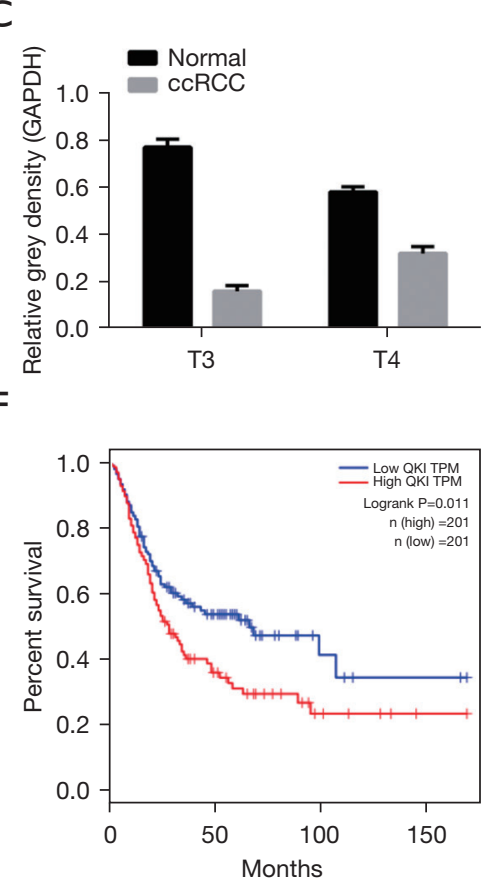

Figure 1 Characteristic of QKI-5 in clear cell renal cell carcinoma (ccRCC). (A) Real-time quantitative reverse transcription polymerase chain reaction (qRT-PCR) was used to detect the expression of QKI-5 in normal and ccRCC tissues ( $\mathrm{n}=20)$. (B,C) Expression of QKI-5 was detected using western blot in normal and kidney renal clear cell carcinoma (KIRC) tissues $(n=6)$ and relative grey density was calculated using image J. Glyceraldehyde 3-phosphate dehydrogenase (GAPDH) acted as a control. (D) Immunohistochemical staining analysis for QKI-5 in primary and metastatic ccRCC tissues $(\mathrm{n}=20 ; \times 200)$. (E,F) Overall survival analysis of QKI-5 expression in ccRCC patients during the $\mathrm{T} 3$ and $\mathrm{T} 4$ periods. Data are shown as mean $\pm \mathrm{SD} .{ }^{* *}, \mathrm{P}<0.01$, ccRCC group $v s$. normal group.

\section{Elevated expression of QKI-5 in ACHN cells compared to HK-2 cells}

To understand the function of QKI-5 in KIRC, the expression of QKI-5 in different kidney cells was investigated using qRT-PCR and western blot. As Figure 2A-2C show, compared with HK-2 cells (human renal tubular epithelial cells), the expression of QKI-5 was significantly elevated in ACHN cell (cell line which was initiated from malignant pleural effusion of metastatic renal adenocarcinoma) while it was downregulated in $\mathrm{SN}$ 12C, SKRC-39, A-498, Caki-2, and 786-0 cells. To assess the expression levels of cell cultures, a suppressing QKI-5 ACHN cell line, an overexpressing the QKI-5 Caki2 cell line, and overexpressing and suppressing QKI-5 786-0 cells were generated and used for further study. As Figure $2 \mathrm{D}-2 \mathrm{H}$ shows, QKI-5 was successfully downregulated in ACHN and 786-0 cells and upregulated in 786-0 and Caki-2 cell lines, suggesting that these cell lines could be used for further analysis.

\section{Upregulation of QKI-5 promotes cell migration and invasion}

To understand the effect of QKI-5 on the progression of KIRC, migration and invasion were detected in 786$0, \mathrm{ACHN}$, and Caki-2 cell lines through overexpressing or suppressing the expression of QKI-5. As Figure $3 A, 3 B$ show, invasion was significantly blocked in the sh-QKI-5-1 group compared with the sh-QKI-5-NC group, while it was enhanced in the Over-QKI-5 group compared with the Over-QKI-5-NC group in 786-0 cells. The migration ability detection results showed that the migration rate of the 786-0 cells was significantly decreased when suppressing QKI-5 expression, while it was dramatically increased when overexpressing QKI-5 expression in 7860 cells (Figure 3C,3D). In addition, downregulation of QKI-5 expression in ACHN cells significantly decreased the invasion and migration ability of ACHN cells, while upregulation of QKI-5 expression in Caki-2 cells significantly elevated the invasion and migration ability of 
A

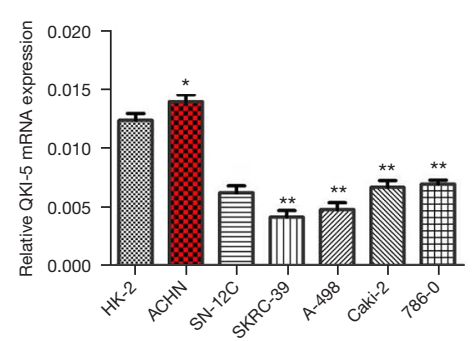

D

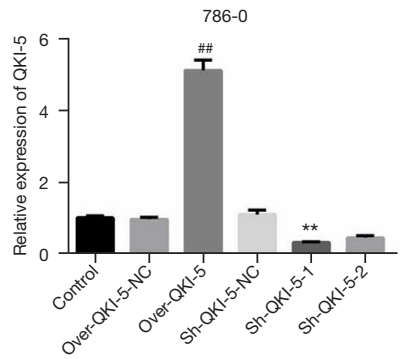

B

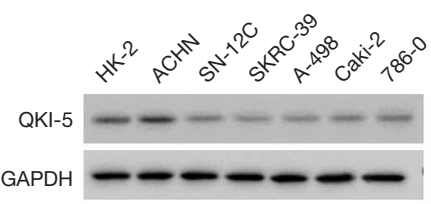

E

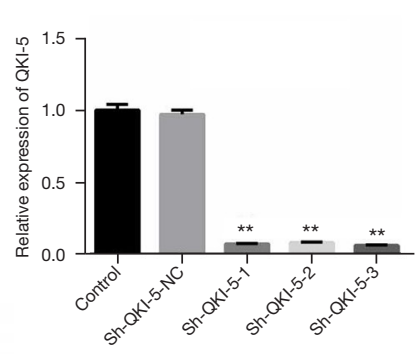

C

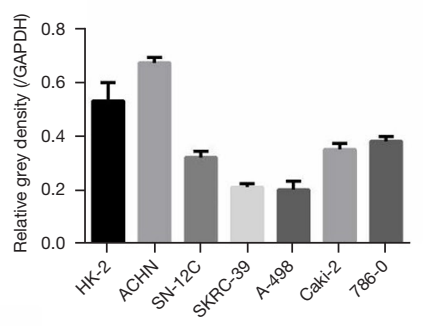

$\mathrm{F}$

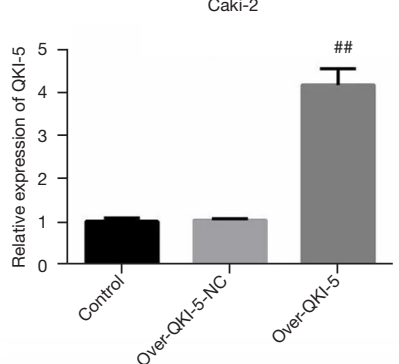

G
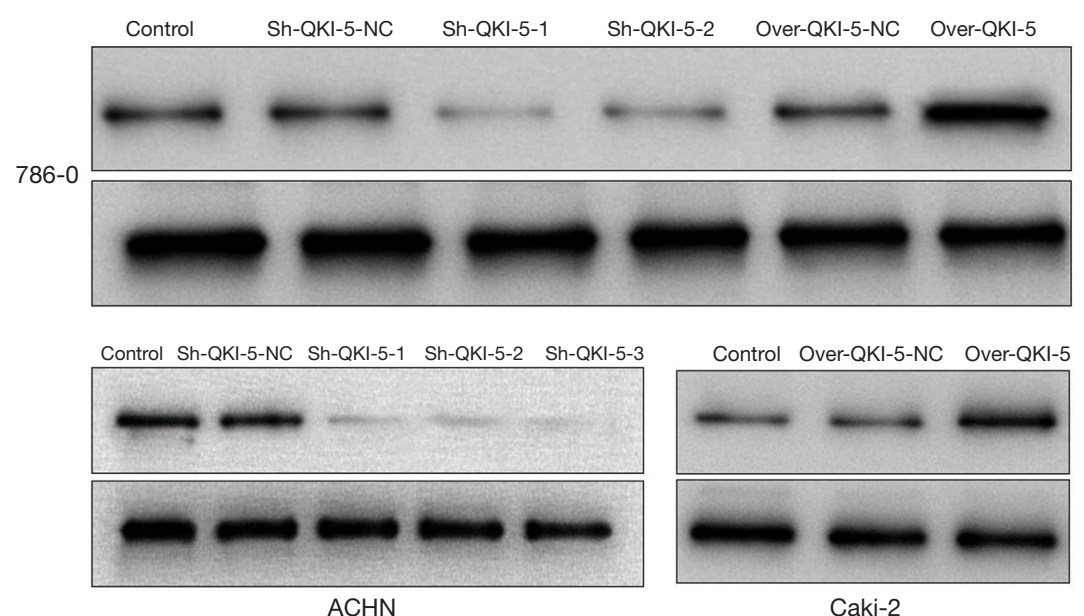

$\mathrm{H}$
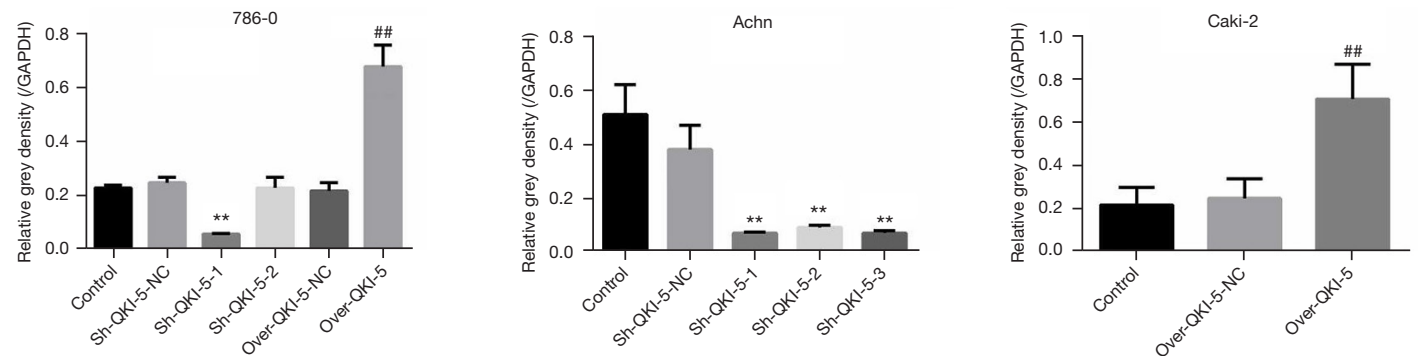

Figure 2 The expression of QKI-5 was elevated in ACHN cells compared to HK-2 cells. (A) Relative expression of QKI-5 in different cell lines detected using real-time quantitative reverse transcription polymerase chain reaction (qRT-PCR), including HK-2, ACHN, SN-12C, SKRC-39, A-498, Caki-2, and 786-0 cell lines; (B) expression of QKI-5 in different cell lines detected using western blot; (C) Relative grey density of QKI-5 were calculated using image J; (D-F) relative expression changes of QKI-5 detected using qRT-PCR in 786-0, ACHN, and Caki-2 cells with QKI-5 overexpression or suppression; (G) Relative expression changes of QKI-5 detected using western blot in 786-0, ACHN, and Caki-2 cells with QKI-5 overexpression or suppression. (H) Relative grey density of QKI-5 were calculated using image J; Data are shown as mean $\pm \mathrm{SD}$, glyceraldehyde 3 -phosphate dehydrogenase (GAPDH) acted as control. *, $\mathrm{P}<0.05 ;{ }^{* *}, \mathrm{P}<0.01$ vs. HK-2 in (A) or vs. sh-QKI-5-NC group in (C,D,G); ${ }^{\#}, \mathrm{P}<0.01$ vs. Over-QKI-5-NC group in (C,E,G). 

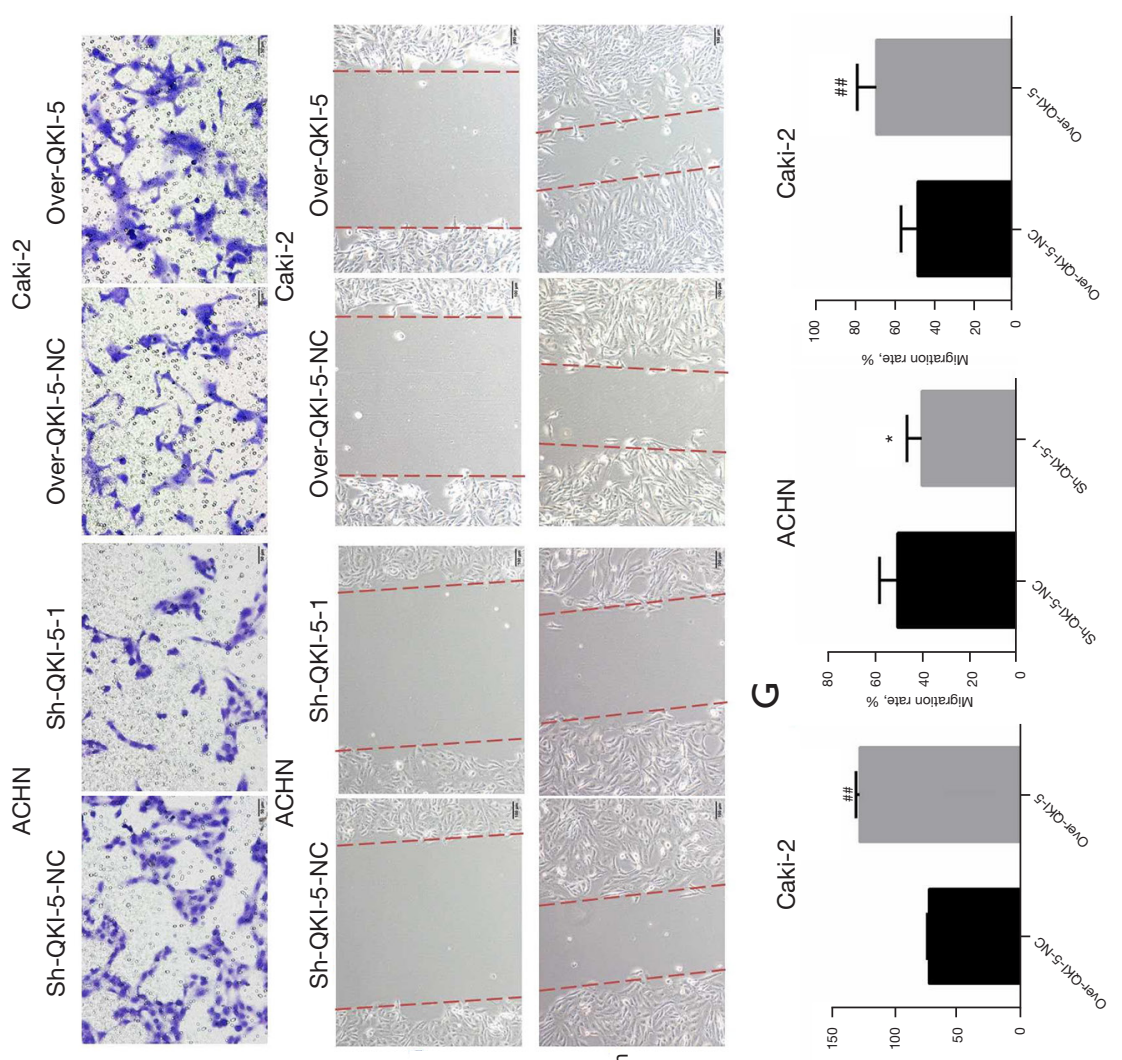

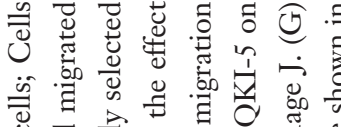

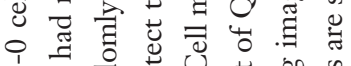

D.

㟧

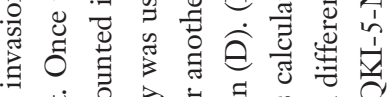

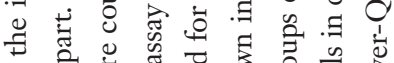

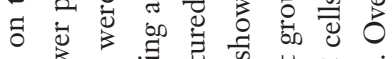

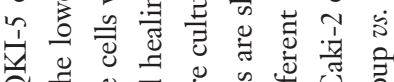

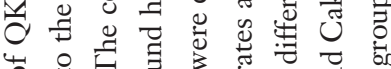

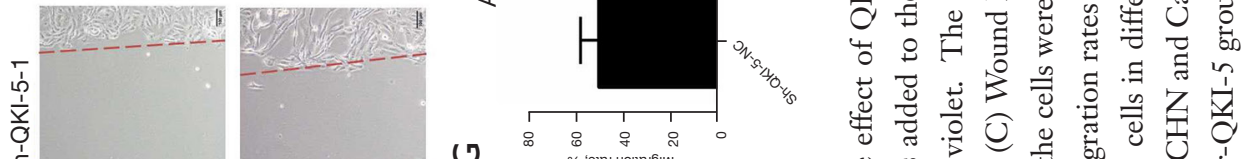

山
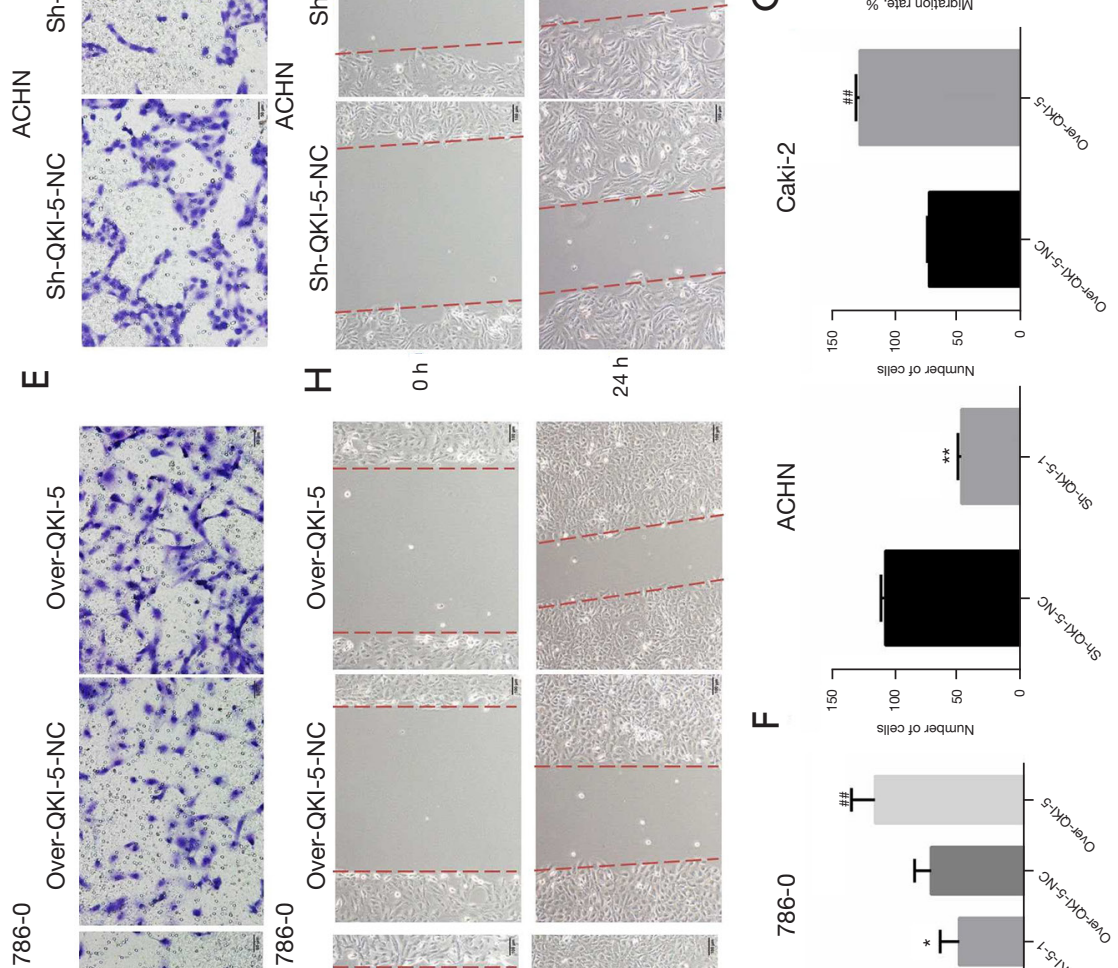

I c 는

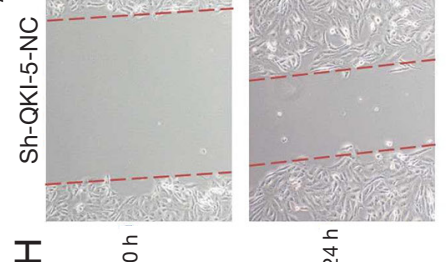

- 5 .

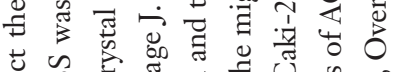

苞会

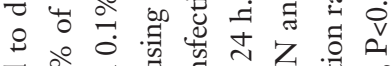

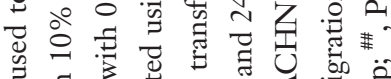

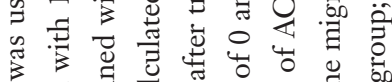

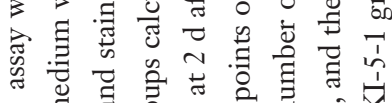

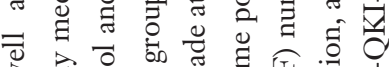

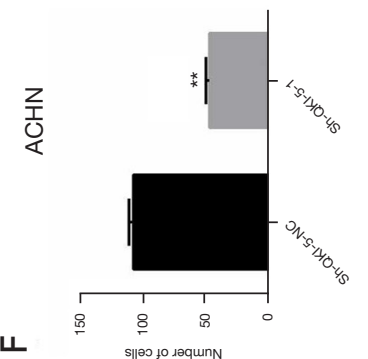

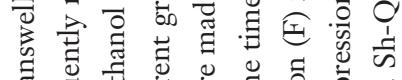

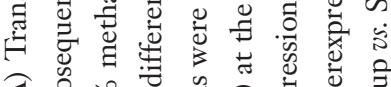

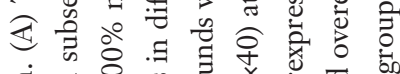

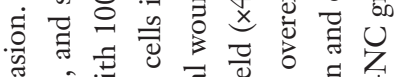

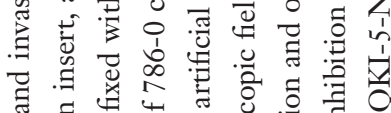

ᄂ

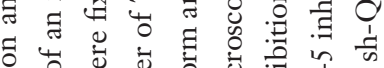

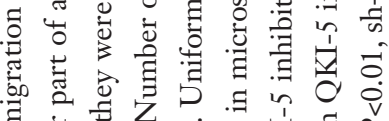

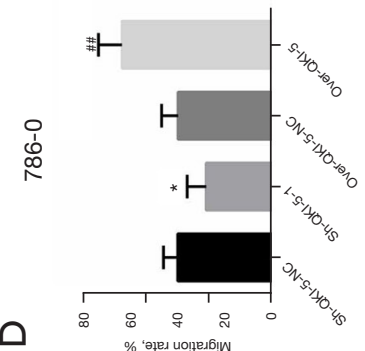

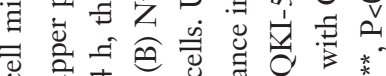

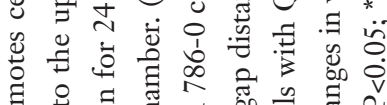
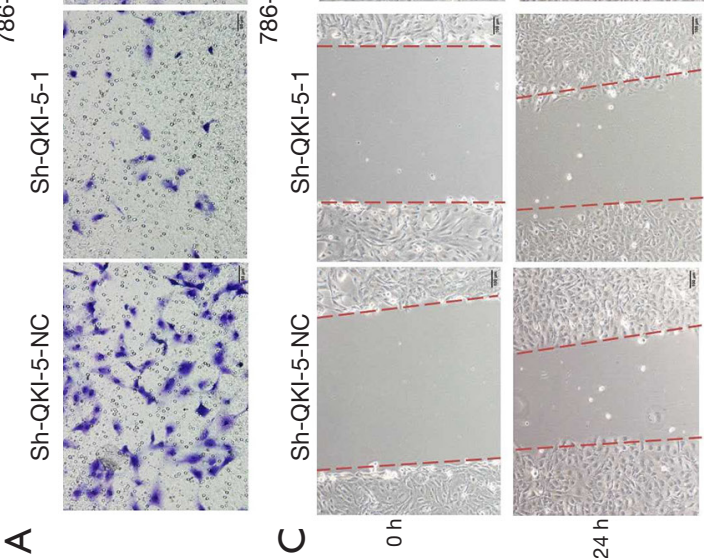

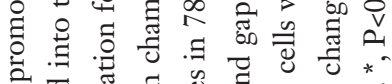

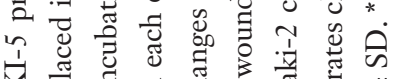

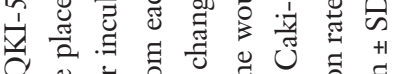

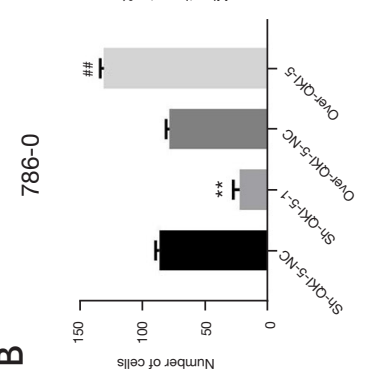

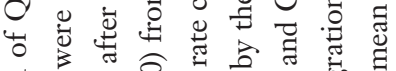

.

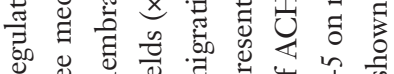

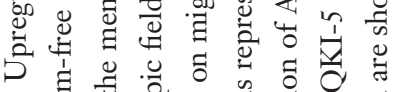

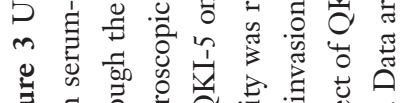

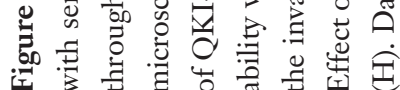


A
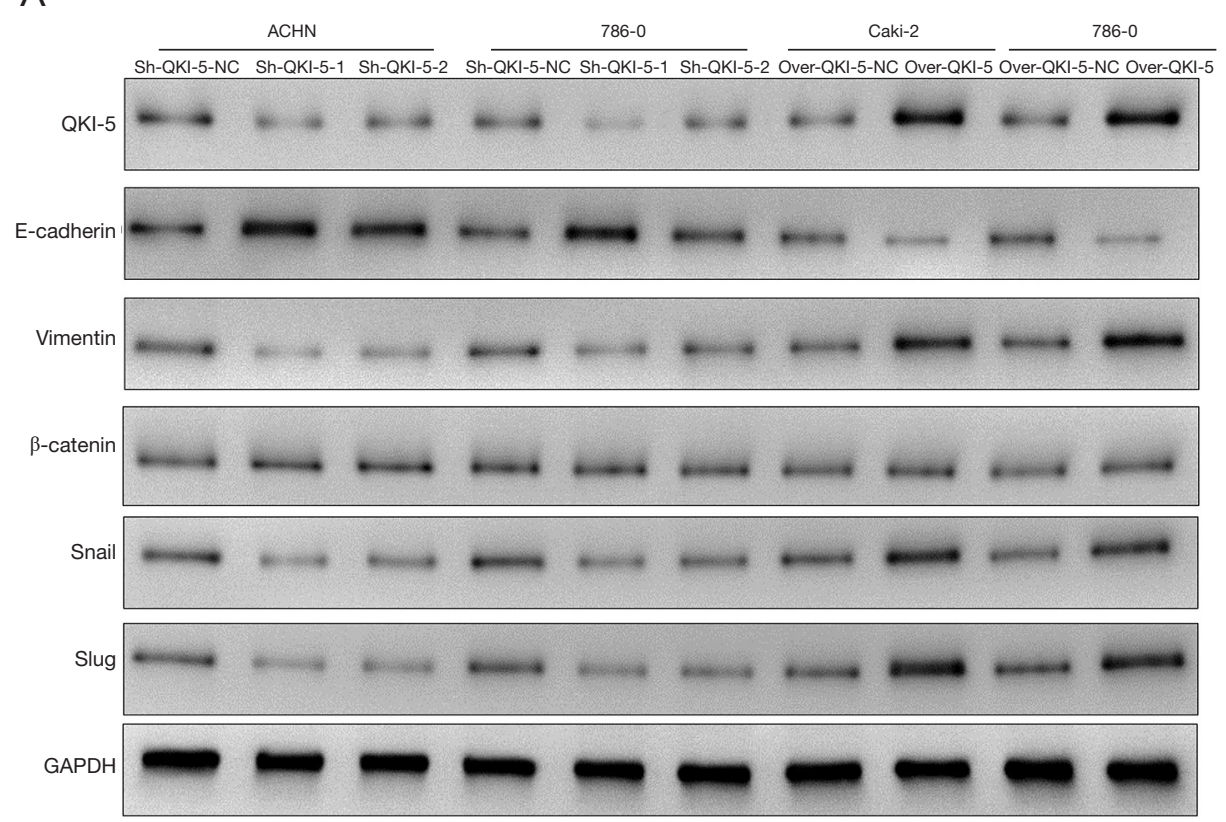

B

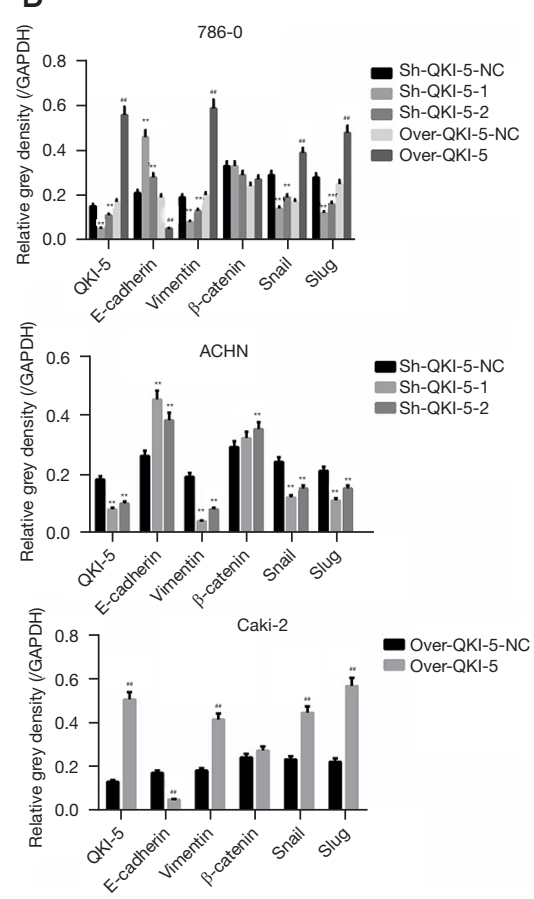

Figure 4 QKI-5 affected the expression of epithelial-mesenchymal transition (EMT) marker genes. (A) Western blot was used to detect the expression of EMT marker genes affected by QKI-5, and relative grey density was calculated using image J. (B) This included E-cadherin, vimentin, $\beta$-catenin, snail and slug. Glyceraldehyde 3-phosphate dehydrogenase (GAPDH) acted as control. Data are shown as mean \pm SD. **, $\mathrm{P}<0.01$, sh-QKI-5-NC group vs. Sh-QKI-5-1 group; ${ }^{\# \#, ~} \mathrm{P}<0.01$, Over-QKI-5 group vs. Over-QKI-5-NC group.

Caki-2 cells (Figure $3 E-3 H$ ). These results demonstrate that upregulation of QKI-5 promotes cell migration and invasion.

\section{QKI-5 affects the expression of EMT marker genes}

It has been demonstrated that QKI-5 participates in the EMT process in several cancers, such as head and neck squamous cell carcinoma (HNSCC). To understand the effect of QKI-5 on the EMT process in KIRC, the expression of several EMT-related genes was investigated, including $\mathrm{E}$-cadherin, vimentin, $\beta$-catenin, snail and slug. The results showed that the expression of QKI-5 was overexpressed in 786-0 and Caki-2 cells and successfully suppressed in 786-0 and ACHN cells (Figure 4A,4B). Further analysis showed that the expression of vimentin snail and slug was significantly increased both in overexpressing 786-0 cells and Caki-2 cells, while it was significantly decreased in suppressing 7860 and ACHN cells. However, the expression changes of E-cadherin showed the opposite results in different cell lines (Figure $4 A, 4 B$ ). In addition, no expression changes of $\beta$-Catenin were observed in different groups (Figure $4 A, 4 B$ ).

\section{QKI-5 was regulated by miR-200c}

MicroRNA regulation of tumor-related genes is one of the most important regulatory patterns in the process of tumor growth. To understand the miRNAs which directly target QKI-5, QKI-5-targeted miRNAs were investigated using Targetscan, Starbase, and miRWalk. In total, from the three databases, 304 common miRNAs were predicted to bind with QKI-5 directly (Figure 5A). Further analysis showed that there were six miRNAs that showed negative correlations with QKI-5 using KIRC data from TCGA. and miR-200c, which showed the most negative correlations, including miR$200 \mathrm{c}(\mathrm{r}=-0.592), \operatorname{miR}-96-5 \mathrm{p}(\mathrm{r}=-0.368), \operatorname{miR}-429(\mathrm{r}=-0.366)$, miR-200b-3p ( $\mathrm{r}=-0.347)$, miR-141-3p ( $\mathrm{r}=-0.320)$, and miR$4784(\mathrm{r}=-0.307)$ (Figure 5B, 5C). Therefore, miR-200c was selected for further analysis. It showed that the expression of miR-200c was significantly upregulated in KIRC tissues compared with normal tissues (Figure 5D). Furthermore, it showed that a binding site (UAAGACA) existed of miR200c in the 3'UTR of QKI-5 (Figure 5E). A dual-luciferase reporter assay showed that compared with the NC (non- 
A
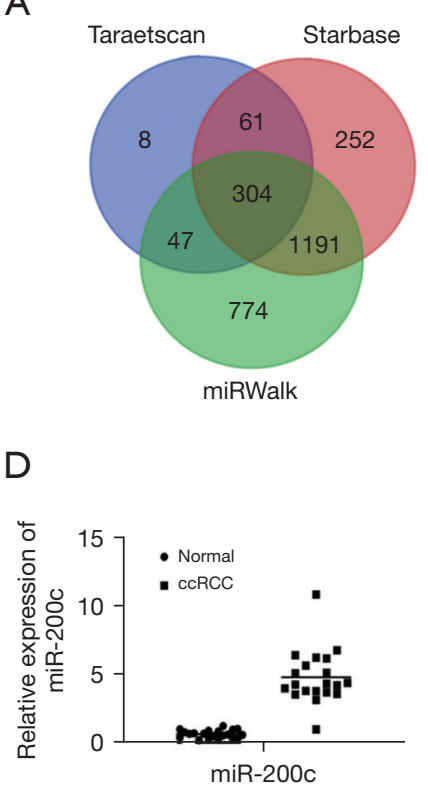

B

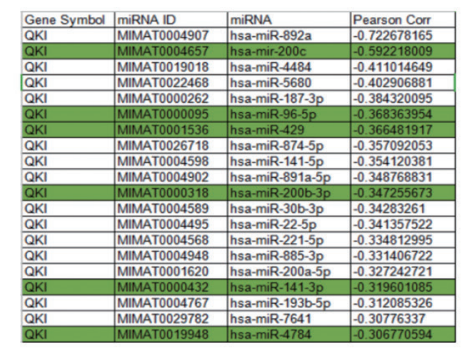

$\mathrm{E}$

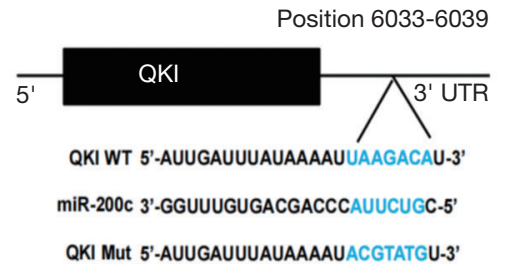

C

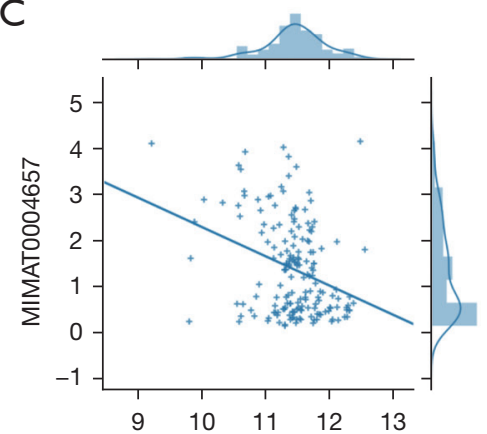

$\mathrm{F}$

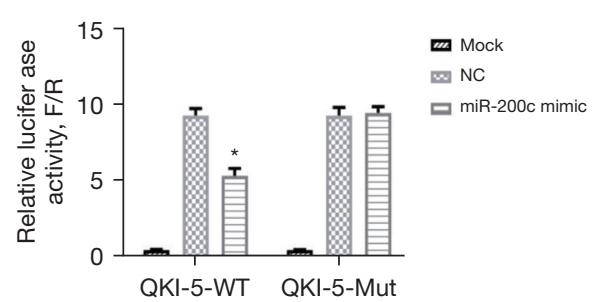

Figure 5 QKI-5 was regulated using microRNA 200c (miR-200c). (A) Target miRNAs of QKI-5 predicted by Targetscan 7.2, Starbase and miRWalk. (B) Top 20 miRNAs showed negative correlations with the expression of QKI-5 from the Cancer Genome Atlas (TCGA). (C) Correlation between the expression of QKI-5 and miR-200c. (D) Relative expression of miR-200c in normal and clear cell renal cell carcinoma (ccRCC) tissues detected by real-time quantitative reverse transcription polymerase chain reaction (qRT-PCR). (E) The binding sites of miR-200c in 3'UTR of QKI-5. (F) Dual-luciferase reporter assay to detect the regulation of miR-200c to QKI-5 by binding with 3'UTR of QKI-5. Glyceraldehyde 3-phosphate dehydrogenase (GAPDH) acted as control. Data are shown as mean $\pm \mathrm{SD}$. *, P<0.05, mir200c group vs. NC group.

specific control) group, the expression of luciferase was significantly inhibited in the QKI-5 wild-type group when supplemented with miR-200c mimic, while there was no significant difference in the expression of luciferase, when the miR-200c binding sites of QKI-5 were mutated (Figure 5F). These results demonstrate that QKI-5 is one of the directly binding targets for miR-200c.

\section{Upregulation of miR-200c suppressed cell invasion and migration in 786-0 and Caki-2 cell lines}

Functional analysis of miR-200c to cell invasion and migration in 786-0 and Caki-2 cell lines showed that upregulation of miR-200c inhibited the invasion and migration ability in 786-0 and Caki-2 cell lines (Figure 6A,6B). In addition, upregulation of $\mathrm{miR}-200 \mathrm{c}$ attenuated the elevation of invasion and migration ability in 786-0 and Caki2 cell lines caused by QKI-5 overexpression (Figure 6C,6D). These results demonstrate that upregulation of miR-200c suppressed cell invasion and migration in 786-0 and Caki-2 cell lines by targeting QKI-5.

\section{Upregulation of miR-200c changes the expression of EMT marker genes}

Western blot analysis showed that the expression of vimentin, snail and slug was significantly decreased, while the expression of E-cadherin was significantly upregulated when miR-200c was added to 786-0 and Caki-2 cells. However, the expression of $\beta$-catenin was not affected by miR-200c mimic (Figure $7 A-7 C$ ). In addition, the expression changes of vimentin, E-cadherin, snail and slug were partially reversed when miR-200c mimics were added to overexpressing QKI-5 786-0 and Caki-2 cells (Figure 7A-7C). The expression changes of vimentin, $\mathrm{E}$-cadherin and $\beta$-catenin in different groups were also confirmed using immunofluorescence analysis in the 786-0 and Caki-2 cells (Figure 7D).

\section{Discussion}

Clear cell renal cell carcinoma is one of the most common types of malignant tumors, and it is difficult to diagnose early and to treat, with high rates of mortality, metastasis 


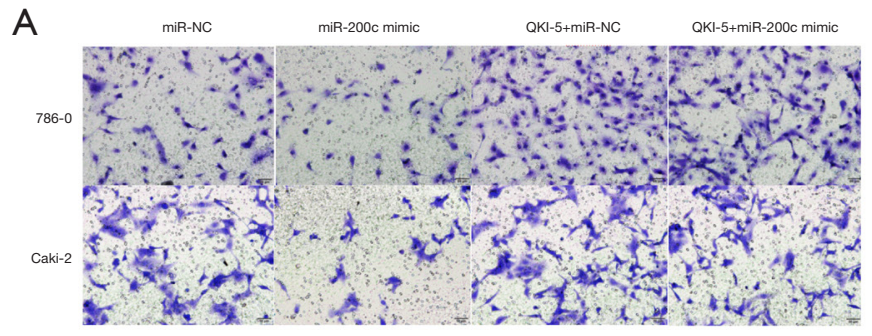

B
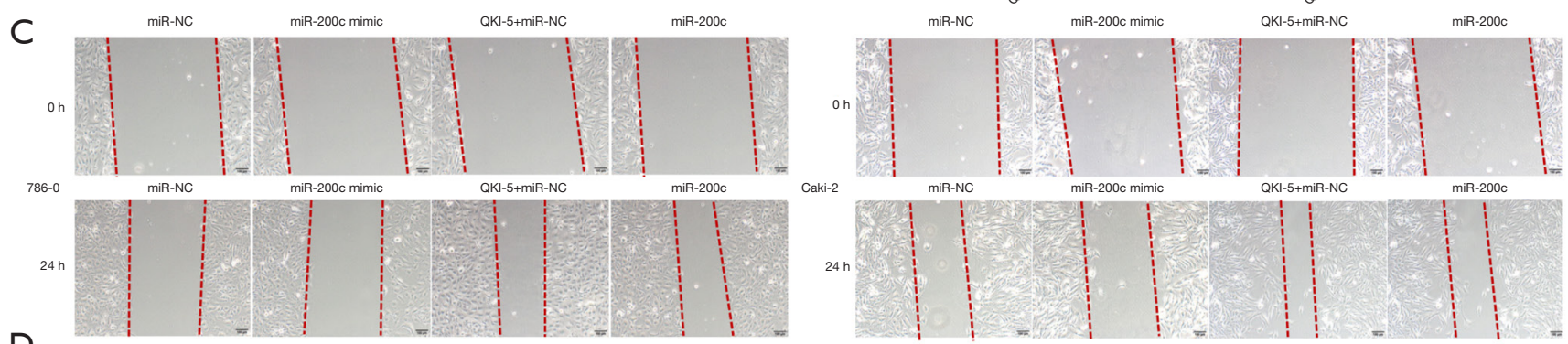

D
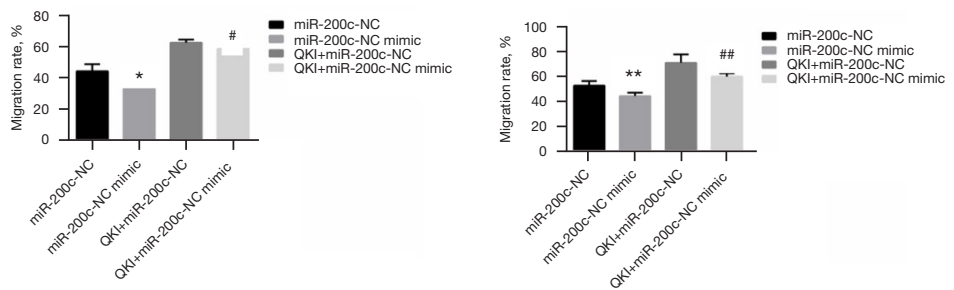

Figure 6 Upregulation of microRNA 200c (miR-200c) suppressed cell invasion and migration in 786-0 and Caki-2 cell lines. (A) Transwell assay was used to detect the effect of microRNA 200c (miR-200c) on the invasion of 786-0 and Caki-2 cell lines (stained with crystal violet, $\times 200$ ); (B) Number of 786-0 and Caki-2 cells in different groups calculated using image J; (C) wound healing - assay was used to detect the effect of miR-200c on migration rate changes in 786-0 and Caki-2 cell lines, Uniform artificial wounds were made at $2 \mathrm{~d}$ after transfection and the cells were cultured for another $24 \mathrm{~h}$. Cell migration ability was represented by the wound gap distance in microscopic field ( $\times 40)$ at the time points of 0 and $24 \mathrm{~h}$. and the migration rates are shown in (D). Data shown as mean \pm SD. **, $<<0.01$, miR-200c-NC mimic group vs. miR-200c-NC group; ${ }^{\#}, \mathrm{P}<0.05$; $^{\#}, \mathrm{P}<0.01$, QKI-5+miR-200c mimic group vs. QKI-5+miR-200c NC group.

and recurrence in patients (21). Although significant progress has been made in the diagnosis and treatment of KIRC, 20-30\% patients present with metastases to the lungs, brain, and other organs $(22,23)$. A large number of genes have been discovered, including noncoding RNAs or signal pathways that are closely related to the occurrence and development of KIRC (24). The protein QKI-5 belongs to the signal transduction and activation of RNA (STAR) protein family, and it is also a key posttranscriptional regulator. It exhibits dual functions in various kinds of cancers by acting as an oncogene or antioncogene. For example, QKI-5 suppresses proliferation of oral squamous cell carcinoma cells via the MAPK and $\beta$-catenin signaling pathways (25). However, upregulation of QKI-5 promotes cell proliferation, migration, and invasion of esophageal squamous cell carcinoma cells (7). It has been demonstrated that in approximately $25 \%$ of KIRC patients the loss of RNA-binding protein QKI at 6q26 (26) is involved, suggesting that QKI plays crucial roles in KIRC. We have demonstrated that QKI-5 is frequently downregulated in KIRC patients, and its downregulation is significantly associated with clinical features including tumor (T) status, metastasis $(\mathrm{M})$ status, differentiation grade, and poorer patient prognosis (9). However, at the same time, once the tumor infiltrates into the extra capsular adipose tissue of kidney, the expression of level of QKI-5 mRNA is significantly higher than that of the adjacent normal tissue. In the present study, further analysis of QKI-5 showed that the expression of QKI-5 is significantly upregulated in metastatic KIRC compared to that in primary foci, and it also shows higher expression in ACHN cells than that in HK-2. We also know that ACHN cells originate from 

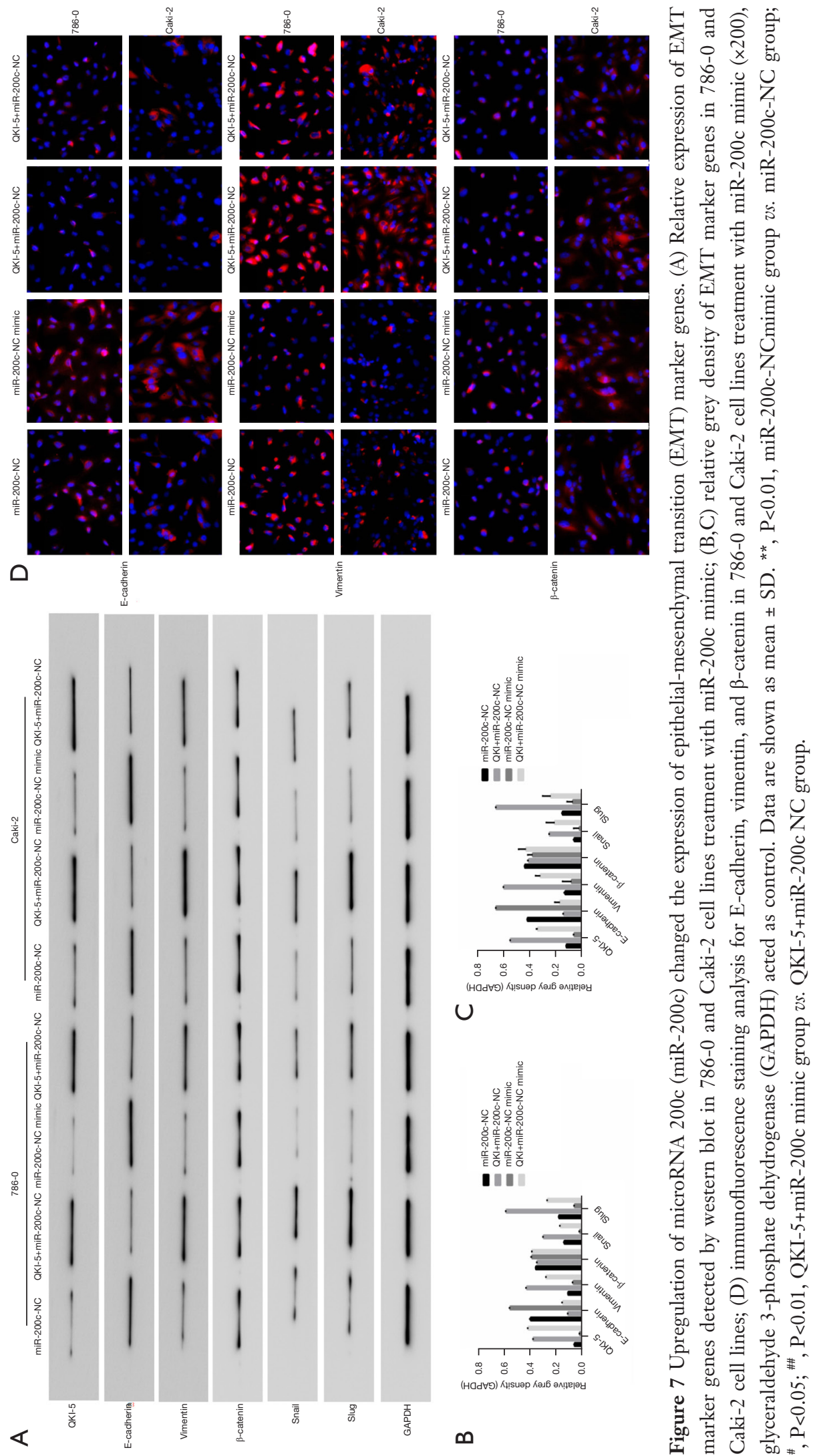
metastatic renal adenocarcinoma, suggesting that QKI-5 might be a key regulator in the metastasis of KIRC. The overall survival analysis of $\mathrm{T} 3$ and $\mathrm{T} 4$ status of KIRC patients showed that lower QKI-5 expression improved overall survival. These results demonstrate that QKI-5 plays important roles in the metastasis of KIRC. However, the exact role of QKI-5 in KIRC metastasis is unknown. In the present study, we showed that overexpressing QKI-5 promotes invasion and migration in 786-0 and Caki-2 cells while suppressing QKI-5-inhibits invasion and migration in 786-0 and ACHN cells, indicating that QKI-5 promotes metastasis of KIRC. Mostly in our opinion, the expression of a gene was downregulated in tumor tissue, and the downregulation of the gene improved migration and invasion, but in our current research, the increased expression QKI-5 promoted cell migration and invasion, we think it's an interesting phenomenon. Maybe it was the gene complicated function in the different stage of the disease, as we know, being an RNA binding protein, QKI-5 can directly bind to and regulates hundreds of alternative splicing targets and exerts pleiotropic effects, such as cell growth, migration and invasion, which deserve our further more research.

MicroRNAs are highly conserved single-stranded non-coding small RNA, which are composed of 19 to 25 nucleotides. The regulatory function of miRNA on the expression of related target genes is mainly achieved through posttranscriptional gene silencing by binding 3'UTR of the target genes. In recent years, miRNAs have been found to be closely associated with the outcome of the patients with KIRC by affecting cancer cell proliferation, differentiation, apoptosis, invasion, and metastasis, such as miR-508, miR$337-3 p$, and miR-663 (27,28). MicroRNA-663 inhibits the proliferation and invasion of clear cell renal cell carcinoma cells by directly targeting PAK4. Several miRNAs have also become potential biomarkers of KIRC such as miR205 and miR-210 (29-31). MicroRNA 200c belongs to the miR-200 family, which has been demonstrated to participate in the development of KIRC via affecting different cell processes and metastasis (32). The miR-200 family has been considered to be a prognostic marker in KIRC (33). In addition, some target genes of miR-200c have also been investigated, including CDK2, CYP1B1, SLC6A1, and heme oxygenase-1 (34-37). Pillman et al. demonstrated that miR-200c and miR-375 regulate epithelial plasticityassociated alternative splicing by repressing QKI-5 (16). Consistent with this result, we showed that miR-200c has a negative correlation with QKI-5 and is a direct target of miR-200c. Further analysis showed that overexpression miR-
$200 \mathrm{c}$ attenuates the effect of invasion and migration caused by QKI-5 both in 786-0 and Caki-2 cells. These results demonstrate that QKI-5 suppression via the regulation of miR-200c inhibits invasion and migration of KIRC.

Deregulated EMT constitutes one of the major aspects of cancer progression. Studies have confirmed that EMT play an important role in tumor invasion and metastasis. During EMT, QKI-5 directly binds to and regulates hundreds of alternative splicing targets, which play crucial roles in tumor growth (13), suggesting QKI-5 can affect metastasis of epithelium-derived cancers. Several studies have demonstrated that QKI-5 can promote the EMT process. For example, MiR-143-3p inhibits cell proliferation, invasion, and EMT by targeting QKI-5 in esophageal squamous cell carcinoma (ESCC) (7). Inhibition of QKI-5 suppresses migration, invasion, and TGF- $\beta 1$-induced EMT of lung cancer cells (5). Consistent with previous studies, in our study, we found that overexpressing QKI-5 can promote the EMT process by elevating the expression of vimentin, snail, slug and downregulating E-cadherin in ccRCC cells. In addition, a number of studies have demonstrated that miR$200 \mathrm{c}$ acts as a tumor suppressor involved in the inhibition of tumor metastases by repressing the EMT process. For example, tamoxifen reverses EMT by demethylating miR200c in triple-negative breast cancer cells (38), and nicotine promotes metastasis and EMT in human colorectal cancer cells by downregulating miR-200c (20). Also, miR-200c has been found to be involved in the process of mesenchymalto-epithelial transition (MET) during renal development. For example, the entire miR-200 seed family is strongly deregulated in ccRCC compared to proximal tubular epithelial cells of the kidney (39). Also, miR-200c modulates the EMT in human renal cell carcinoma metastasis by affecting the Akt-miR-200c-E-cadherin axis (40). In the present study, we found that miR-200c attenuates the effect of QKI-5 on the expression of vimentin, snail, slug and E-cadherin, demonstrating that suppression of QKI-5 inhibited the EMT process via regulation by miR-200c. Taken together, we demonstrated that QKI-5 inhibited by miR-200c attenuated invasion and migration via inhibiting the EMT process in KIRC. However, more systematic clinical experiments need to be designed and performed to provide a reliable basis for clinical diagnosis and targeted therapy of KIRC.

\section{Conclusions}

In summary, the present study revealed that suppressing QKI-5 inhibits invasion and migration of KIRC via inhibiting 
EMT transition regulated by miR-200c, suggesting that QKI-5 may be a potential marker and therapeutic target of renal clear cell carcinoma metastasis.

\section{Acknowledgments}

Funding: This work was supported by the National Natural Science Foundation of China (grant number 81760452).

\section{Footnote}

Reporting Checklist: The authors have completed the MDAR reporting checklist. Available at https://dx.doi. org/10.21037/tau-21-833

Data Sharing Statement: Available at https://dx.doi. org/10.21037/tau-21-833

Conflicts of Interest: All authors have completed the ICMJE uniform disclosure form (available at https://dx.doi. org/10.21037/tau-21-833). All authors reported this work was supported by the National Natural Science Foundation of China (No. 81760452). The authors have no other conflicts of interest to declare.

Ethical Statement: The authors are accountable for all aspects of the work in ensuring that questions related to the accuracy or integrity of any part of the work are appropriately investigated and resolved. All procedures performed in this study involving human participants were in accordance with the Declaration of Helsinki (as revised in 2013). The project has been approved by the ethics committee of the First Affiliated Hospital of Xinjiang Medical University (No.: 20180213-09), and informed consent was taken from all the patients.

Open Access Statement: This is an Open Access article distributed in accordance with the Creative Commons Attribution-NonCommercial-NoDerivs 4.0 International License (CC BY-NC-ND 4.0), which permits the noncommercial replication and distribution of the article with the strict proviso that no changes or edits are made and the original work is properly cited (including links to both the formal publication through the relevant DOI and the license). See: https://creativecommons.org/licenses/by-nc-nd/4.0/.

\section{References}

1. Linehan WM, Ricketts CJ. The Cancer Genome Atlas of renal cell carcinoma: findings and clinical implications. Nat Rev Urol 2019;16:539-52.

2. Cheng SK, Chuah KL. Metastatic Renal Cell Carcinoma to the Pancreas: A Review. Arch Pathol Lab Med 2016;140:598-602.

3. Vermassen T, De Meulenaere A, Van de Walle M, et al. Therapeutic approaches in clear cell and non-clear cell renal cell carcinoma. Acta Clin Belg 2017;72:12-8.

4. Wu M, Huang Y, Chen T, et al. LncRNA MEG3 inhibits the progression of prostate cancer by modulating miR-95p/QKI-5 axis. J Cell Mol Med 2019;23:29-38.

5. Zhou X, Li X, Sun C, et al. Quaking-5 suppresses aggressiveness of lung cancer cells through inhibiting -catenin signaling pathway. Oncotarget 2017;8:82174-84.

6. Kim EJ, Kim JS, Lee S, et al. QKI, a miR-200 target gene, suppresses epithelial-to-mesenchymal transition and tumor growth. Int J Cancer 2019;145:1585-95.

7. He Z, Yi J, Liu X, et al. MiR-143-3p functions as a tumor suppressor by regulating cell proliferation, invasion and epithelial-mesenchymal transition by targeting QKI5 in esophageal squamous cell carcinoma. Mol Cancer 2016;15:51.

8. Azam SH, Porrello A, Harrison EB, et al. Quaking orchestrates a post-transcriptional regulatory network of endothelial cell cycle progression critical to angiogenesis and metastasis. Oncogene 2019;38:5191-210.

9. Zhang RL, Yang JP, Peng LX, et al. RNA-binding protein QKI-5 inhibits the proliferation of clear cell renal cell carcinoma via post-transcriptional stabilization of RASA1 mRNA. Cell Cycle 2016;15:3094-104.

10. Chen T, You Y, Jiang H, et al. Epithelial-mesenchymal transition (EMT): A biological process in the development, stem cell differentiation, and tumorigenesis. J Cell Physiol 2017;232:3261-72.

11. Mittal V. Epithelial Mesenchymal Transition in Tumor Metastasis. Annu Rev Pathol 2018;13:395-412.

12. Dongre A, Weinberg RA. New insights into the mechanisms of epithelial-mesenchymal transition and implications for cancer. Nat Rev Mol Cell Biol 2019;20:69-84.

13. Yang Y, Park JW, Bebee TW, et al. Determination of a Comprehensive Alternative Splicing Regulatory Network and Combinatorial Regulation by Key Factors during the Epithelial-to-Mesenchymal Transition. Mol Cell Biol 2016;36:1704-19.

14. Liang G, Meng W, Huang X, et al. miR-196b-5pmediated downregulation of TSPAN12 and GATA6 promotes tumor progression in non-small cell lung cancer. 
Proc Natl Acad Sci U S A 2020;117:4347-57.

15. Ma DL, Li JY, Liu YE, et al. Influence of continuous intervention on growth and metastasis of human cervical cancer cells and expression of RNAmiR-574-5p. J Biol Regul Homeost Agents 2016;30:91-102.

16. Pillman KA, Phillips CA, Roslan S, et al. miR-200/375 control epithelial plasticity-associated alternative splicing by repressing the RNA-binding protein Quaking. EMBO J 2018;37:e99016.

17. Mukohyama J, Isobe $\mathrm{T}, \mathrm{Hu} \mathrm{Q}$, et al. miR-221 Targets QKI to Enhance the Tumorigenic Capacity of Human Colorectal Cancer Stem Cells. Cancer Res 2019;79:5151-8.

18. O'Brien SJ, Carter JV, Burton JF, et al. The role of the miR-200 family in epithelial-mesenchymal transition in colorectal cancer: a systematic review. Int J Cancer 2018;142:2501-11.

19. Basu S, Chaudhary A, Chowdhury P, et al. Evaluating the role of hsa-miR-200c in reversing the epithelial to mesenchymal transition in prostate cancer. Gene 2020;730:144264.

20. Lei $Z$, Xiaomin $Y, H e H$, et al. Nicotine downregulates microRNA-200c to promote metastasis and the epithelialmesenchymal transition in human colorectal cancer cells. J Cell Physiol 2019;234:1369-79.

21. Ghatalia P, Rathmell WK. Systematic Review: ClearCode 34 - A Validated Prognostic Signature in Clear Cell Renal Cell Carcinoma (ccRCC). Kidney Cancer 2018;2:23-9.

22. Li QK, Pavlovich CP, Zhang H, et al. Challenges and opportunities in the proteomic characterization of clear cell renal cell carcinoma (ccRCC): A critical step towards the personalized care of renal cancers. Semin Cancer Biol 2019;55:8-15.

23. Sankin A, Hakimi AA, Hsieh JJ, et al. Metastatic nonclear cell renal cell carcinoma: an evidence based review of current treatment strategies. Front Oncol 2015;5:67.

24. Frew IJ, Moch H. A clearer view of the molecular complexity of clear cell renal cell carcinoma. Annu Rev Pathol 2015;10:263-89.

25. Fu X, Feng Y. QKI-5 suppresses cyclin D1 expression and proliferation of oral squamous cell carcinoma cells via MAPK signalling pathway. Int J Oral Maxillofac Surg 2015;44:562-7.

26. Cancer Genome Atlas Research Network. Comprehensive molecular characterization of clear cell renal cell carcinoma. Nature 2013;499:43-9.

27. Wang $\mathrm{W}, \mathrm{Hu} \mathrm{W}$, Wang $\mathrm{Y}$, et al. MicroRNA-508 is downregulated in clear cell renal cell carcinoma and targets ZEB1 to suppress cell proliferation and invasion. Exp Ther Med 2019;17:3814-22.

28. Zhuang Q, Shen J, Chen Z, et al. MiR-337-3p suppresses the proliferation and metastasis of clear cell renal cell carcinoma cells via modulating Capn4. Cancer Biomark 2018;23:515-25.

29. Crentsil VC, Liu H, Sellitti DF. Comparison of exosomal microRNAs secreted by 786-O clear cell renal carcinoma cells and HK-2 proximal tubule-derived cells in culture identifies microRNA-205 as a potential biomarker of clear cell renal carcinoma. Oncol Lett 2018;16:1285-90.

30. Wang X, Wang T, Chen C, et al. Serum exosomal miR210 as a potential biomarker for clear cell renal cell carcinoma. J Cell Biochem 2018. (Epub ahead of print). doi: $10.1002 / j c b .27347$.

31. Zhang W, Ni M, Su Y, et al. MicroRNAs in Serum Exosomes as Potential Biomarkers in Clear-cell Renal Cell Carcinoma. Eur Urol Focus 2018;4:412-9.

32. Jiang J, Yi BO, Ding S, et al. Demethylation drug 5-Aza-2'-deoxycytidine-induced upregulation of miR200c inhibits the migration, invasion and epithelialmesenchymal transition of clear cell renal cell carcinoma in vitro. Oncol Lett 2016;11:3167-72.

33. Saleeb R, Kim SS, Ding Q, et al. The miR-200 family as prognostic markers in clear cell renal cell carcinoma. Urol Oncol 2019;37:955-63.

34. Gao C, Peng FH, Peng LK. MiR-200c sensitizes clearcell renal cell carcinoma cells to sorafenib and imatinib by targeting heme oxygenase-1. Neoplasma 2014;61:680-9.

35. Wang X, Chen X, Han W, et al. miR-200c Targets CDK2 and Suppresses Tumorigenesis in Renal Cell Carcinoma. Mol Cancer Res 2015;13:1567-77.

36. Chang I, Mitsui Y, Fukuhara S, et al. Loss of miR-200c up-regulates CYP1B1 and confers docetaxel resistance in renal cell carcinoma. Oncotarget 2015;6:7774-87.

37. Maolakuerban N, Azhati B, Tusong H, et al. MiR-200c$3 p$ inhibits cell migration and invasion of clear cell renal cell carcinoma via regulating SLC6A1. Cancer Biol Ther 2018;19:282-91.

38. Wang Q, Cheng Y, Wang Y, et al. Tamoxifen reverses epithelial-mesenchymal transition by demethylating miR200c in triple-negative breast cancer cells. BMC Cancer 2017;17:492.

39. Duns G, van den Berg A, van Dijk MC, et al. The entire miR-200 seed family is strongly deregulated in clear cell renal cell cancer compared to the proximal tubular epithelial cells of the kidney. Genes Chromosomes Cancer 2013;52:165-73. 
40. Wang X, Chen X, Wang R, et al. microRNA-200c modulates the epithelial-to-mesenchymal transition in human renal cell carcinoma metastasis. Oncol Rep
2013;30:643-50.

(English Language Editor: B. Meiser)

Cite this article as: Zhang $\mathrm{R}$, Wang W, Aimudula A, Lu S, Lu P, Aihaiti R, Bao Y. Quaking I-5 protein inhibits invasion and migration of kidney renal clear cell carcinoma via inhibiting epithelial-mesenchymal transition suppression through the regulation of microRNA 200c. Transl Androl Urol 2021;10(10):3800-3814. doi: 10.21037/tau-21-833 
Supplementary

Table S1 The sequences of miR-200c mimics and mimics NC

\begin{tabular}{ll}
\hline Primer & 5'-3' \\
\hline MicroRNA (miR)-200c mimics sense & CGUCUUACCCAGCAGUGUUUGG \\
Antisense & CCAAACACUGCUGGGUAAGACG \\
Mimics NC sense & UCACAACCUCCUAGAAAGAGUAGA \\
Antisense & UCUACUCUUUCUAGGAGGUUGUGA \\
\hline
\end{tabular}

